\title{
Correction to: Correlation between incidental fat deposition in the liver and pancreas in asymptomatic individuals
}

\author{
Mounes Aliyari Ghasabeh ${ }^{1} \cdot$ Mohammadreza Shaghaghi $^{1} \cdot$ Pegah Khoshpouri $^{1} \cdot$ Li pan $^{2} \cdot$ Ankur Pandey $^{1}$. \\ Pallavi Pandey ${ }^{1} \cdot$ Xiaodong Zhong $^{3} \cdot$ Stephan Kannengiesser ${ }^{4} \cdot$ Ihab R. Kamel $^{1,5}$
}

Published online: 15 November 2019

(c) Springer Science+Business Media, LLC, part of Springer Nature 2019

\section{Correction to: Abdominal Radiology \\ https://doi.org/10.1007/s00261-019-02206-w}

Unfortunately the article was published with a spell error in the co-author names "Ankur Pandy and Pallavi Pandy". The correct co-author names should be Ankur Pandey and Pallavi Pandey".

Publisher's Note Springer Nature remains neutral with regard to jurisdictional claims in published maps and institutional affiliations.

The original article can be found online at https://doi.org/10.1007/ s00261-019-02206-w.

Ihab R. Kamel

ikamel@jhmi.edu

1 The Russell H. Morgan Department of Radiology and Radiological Sciences, Johns Hopkins University School of Medicine, Baltimore, MD, USA

2 Siemens Healthcare, Baltimore, MD, USA

3 Siemens Healthcare, Atlanta, GA, USA

4 Siemens Healthcare GmbH, Erlangen, Germany

5 The Russell H. Morgan Department of Radiology and Radiological Science, Johns Hopkins Hospital, 600 North Wolfe Street, MRI 143, Baltimore, MD 21287, USA 